\title{
Peripheral T-cell lymphoma unspecified type presenting with a pneumothorax as the initial manifestation: A case report and literature review
}

\author{
TIANHONG FU ${ }^{1 *}$, WEI YANG ${ }^{2 *}$, XUEJIN ZHANG ${ }^{2}$ and XIAOFENG $\mathrm{XU}^{2}$ \\ Divisions of ${ }^{1}$ Pathology and ${ }^{2}$ Hematology, Hangzhou Red Cross Hospital, Hangzhou, Zhejiang 310003, P.R. China
}

Received February 28, 2015; Accepted January 13, 2016

DOI: $10.3892 / \mathrm{ol} .2016 .4518$

\begin{abstract}
The present study describes the case of a 51-year-old male presenting with a pneumothorax, in whom a final diagnosis of peripheral T-cell lymphoma unspecified type (PTCL-u) was determined. As the patient had experienced a shortness of breath subsequent to exercise for a period of 2 months, a fiber bronchoscopy was performed; however, no abnormalities were initially identified. Following the bronchoscopy, the patient suffered a recurrent pneumothorax with symptoms of emaciation, night sweats and a fever. The combination of computed tomography and interstitial lung pathology results suggested a high probability of malignant lymphoma. Further test results confirmed this diagnosis, including the fiber bronchoscopy biopsy report, which indicated a large amount of lymphocyte aggregation, and an ultrasound that identified several supraclavicular and left submandibular enlarged lymph nodes (largest size, $2.3 \times 1.3 \mathrm{~cm}$ ), with an absent hilar lymph node. A biopsy of the left cervical lymph node was performed, with post-operative pathological analysis confirming a diagnosis of peripheral T-cell lymphoma unspecified (stage IVB). The patient received two cycles of CHOP chemotherapy, and the symptoms alleviated following completion of the treatment, with chest radiography indicating that the pneumothorax had disappeared. The patient subsequently refused further treatment due to financial difficulty, and succumbed to the disease 6 months later.
\end{abstract}

\section{Introduction}

Peripheral T-cell lymphoma (PTCL) accounts for $12 \%$ of all non-Hodgkin lymphoma (NHL) cases (1), and includes peripheral T-cell lymphoma unspecified (PTCL-u), anaplastic

Correspondence to: Dr Xiaofeng Xu, Division of Hematology, Hangzhou Red Cross Hospital, 208 Huancheng Road, Hangzhou, Zhejiang 310003, P.R. China

E-mail: hhxuxiaofeng@126.com

${ }^{*}$ Contributed equally

Key words: pneumothorax, peripheral T-cell lymphoma unspecified large cell lymphoma (ALCL) and angioimmunoblastic T-cell lymphoma (AITL) (1). The most common subtype is PTCL-u (1).

As PTCL is extremely invasive, the majority of cases are not associated with a positive prognosis (2). To date, no reliable therapeutic schedule has been established worldwide (3). Currently, clinicians typically refer patients with B-cell lymphoma for CHOP or CHOP-like treatment regimens; however, the cure rate is not particularly high (4). More rigorous chemotherapy schemes, including HyperCVAD and stem cell transplantation, have been tested by several clinics, however, no markedly positive effects have been reported (3), and the side effects from such treatments are detrimental $(5,6)$, thus inducing patient suffering and commonly disease relapse (7). The overall 5-year survival rate for patients with PTCL is $20-30 \%(8,9)$. PTCL has no specific clinical features. The median age of onset is 60 years, and the disease is more prevalent in males, who often present with lymphadenopathy and/or extranodal disease (10). Onset of disease is common within the lymph nodes and also in extranodal regions, which includes the gastrointestinal tract, nasal cavity, sinuses, nasopharynx, oropharynx, skin, tonsils, spleen, liver and bone marrow. The majority of cases are diagnosed during stages III-IV (10). Approximately half of patients present with systemic symptoms, including night sweat, fever, itchy skin and weight loss $(11,12)$.

When air or other gases enter the pleural cavity, this results in a pneumothorax (13). This situation may occur spontaneously, however, the majority of cases are induced by trauma or surgery to the lung or chest wall $(14,15)$. They may also be induced as a result of other diseases, improper diagnosis or improper handling of treatment (9). The incidence rate of primary spontaneous pneumothorax (PSP) for males is 7.4-18 per 100,000 individuals per year, while for females is the incidence is $1.2-6$ per 100,000 individuals $(15,16)$. The clinical behavior of PSP presents as an acute onset of local pleuritic pain associated with short breath at rest (17). This pain may be mild, severe, sharp or a steady ache, but can usually be resolved within $24 \mathrm{~h}$ even if a pneumothorax still exists (15). When a large pneumothorax occurs, defined as when free air occupies more than $15-20 \%$ of the hemithorax, decreased breath sounds on auscultation, reduced chest wall movement, hyper-resonance (tympanic) on percussion and reduced tactile fremitus on palpation of the chest may often 


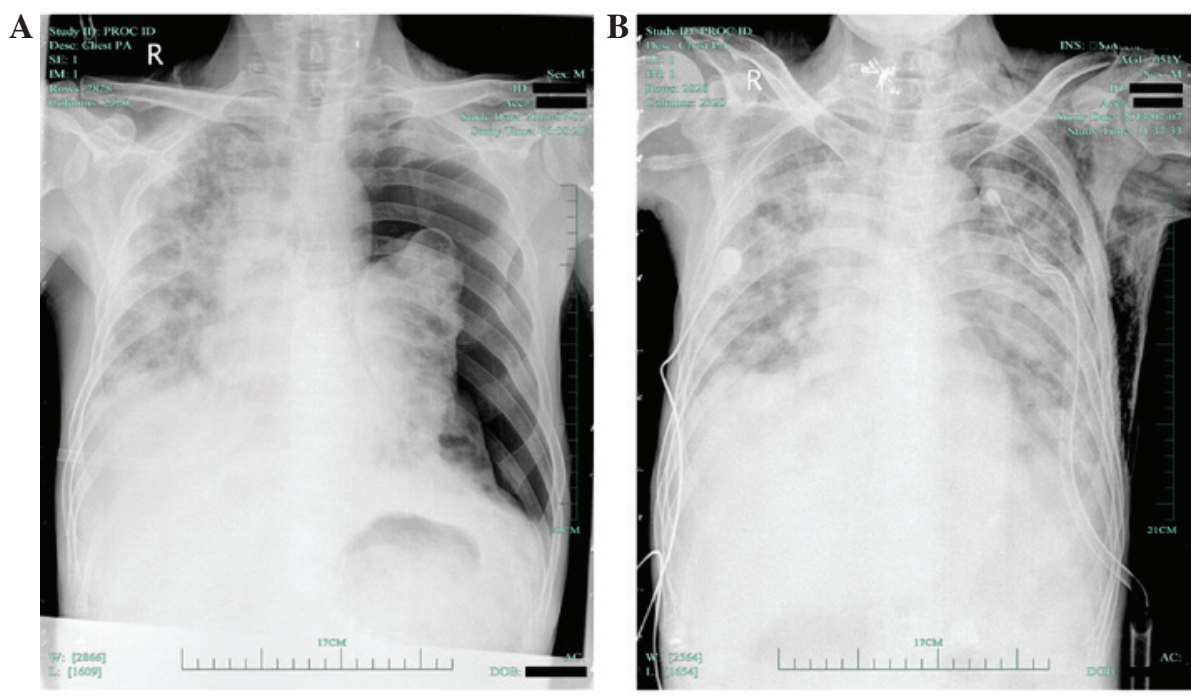

Figure 1. Chest X-ray images of the (A) left pneumothorax and (B) recovery of the left pneumothorax.

be detected during clinical diagnosis (14). If patients experience discomfort in the form of circulatory or respiratory compromise, this is often a result of reflex tachycardia (13). This will induce rapid pulmonary re-expansion following decompression, which is life-threatening (18). Pneumothorax is frequently induced by pulmonary inflammation, tuberculosis and tumors (15). Clinically, pulmonary lymphomas rarely induce a pneumothorax, and a very limited number of relevant reports are available in the literature. Primary and secondary pulmonary lymphomas are predominantly B-cell lymphomas, with only one case of primary pneumothorax as a result of B-cell lymphoma identified in the current literature (19). Pulmonary T-cell lymphoma is particularly rare, and no PTCL-u cases presenting with a pneumothorax as the primary manifestation have been reported. The current study describes a case of PTCL-u with pulmonary infiltration that resulted in a pneumothorax. In addition, a systematic review of the relevant literature is presented with the aim of aiding clinicians in the diagnosis and treatment of PTCL-u pulmonary infiltration.

\section{Case report}

A 51-year-old male was admitted to the Department of Pneumology at the Hangzhou Red Cross Hospital (Hangzhou, China) on June 27, 2013, presenting with a 2-month history of shortness of breath after exercise. At 2 months prior to referral, the patient had visited a local hospital for consultation. A computed tomography (CT; Philips Brilliance 16 Slice; Philips Medical Systems, Inc., Bothell, WA, USA) scan of the chest was performed and the results indicated the possibility of interstitial pneumonia. The patient was subsequently treated with oral clarithromycin; however, the symptoms did not improve and were instead exacerbated. The patient was therefore hospitalized at the Hangzhou Red Cross Hospital.

Physical examination indicated the following: No evidence of mucosal xanthochromia or superficial enlargement of lymph nodes palpable in the systemic skins, slight cyanosis of the lips, a symmetric thoracic cage, and Velcro rales audible under the arm and subscapularis bilaterally. The patient had a heart rate of $72 \mathrm{bpm}$, with a regular heart rhythm, low heart sounds and no pathological murmur. Upon palpation, the patient had a soft abdomen, with no pressing pain or rebound tenderness, no coastal liver or spleen, and no edema in the bilateral lower limbs. The patient was bilateral Babinski sign-negative and all 10 fingers were slightly clubbed. Auxiliary examination revealed the following: White blood

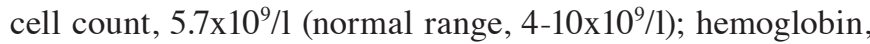
$119 \mathrm{~g} / 1$ (normal range, $10-16 \mathrm{~g} / \mathrm{l}$ ); platelet count, 203x10 $/ 1$ (normal range, 100-300×10 $/ 1$ ); prothrombin time, $10.8 \mathrm{sec}$ (normal range, 10.5-14.3 sec); activated partial thromboplastin time, $31.4 \mathrm{sec}$ (normal range, 18-34 sec); fibrinogen, $207 \mathrm{mg} / \mathrm{dl}$ (normal range, 170-500 mg/dl); D-dimer, $621.0 \mu \mathrm{g} / 1$ (normal range, $0-750 \mu \mathrm{g} / \mathrm{dl}$ ); alanine aminotransferase and creatinine, normal; lactate dehydrogenase, $217 \mathrm{U} / 1$ (normal range, 60-245 U/1); hepatitis B surface antibody, positive; and normal carcinoembryonic antigen, $\alpha$-fetoprotein and cancer antigen 125 levels. On July 2, 2013, the patient underwent a fiber bronchoscopy; as no abnormalities were initially observed, a biopsy was subsequently performed.

On July 5, 2013, the patient experienced sudden chest pain and shortness of breath, and chest radiography indicated the presence of a left pneumothorax (Fig. 1A). The patient underwent closed thoracic drainage and the symptoms gradually improved. On July 7, 2013, an X-ray (OpiTop 150/40/80HC-100 3PH; Siemens AG, Munich, Germany) of the chest demonstrated that the left pneumothorax had nearly recovered (Fig. 1B). However, a chest CT scan (Fig. 2) performed on July 8, 2013, indicated that the right lung and left lung had compressed by $5 \%$ and $60 \%$, respectively, in addition to interstitial inflammation of each lung and pleural pathological changes, including pleural thickening and a small amount of pleural effusion (Fig. 2A). Following several air extractions, the condition of the patient stabilized. Chest CT was repeated on August 5, 2013, and revealed interstitial inflammation of each lung with left pleural effusion; the right lung had now compressed by $20 \%$ (Fig. 2B). Furthermore, the body temperature of the patient continued to increase during hospitalization. Following anti-infective treatment with cefepime (1.0 g, ivgtt q12 h, 7 days) and teicoplanin (400 mg, ivgtt qd, 7 days), the symptoms of fever had not alleviated; however, the 
A
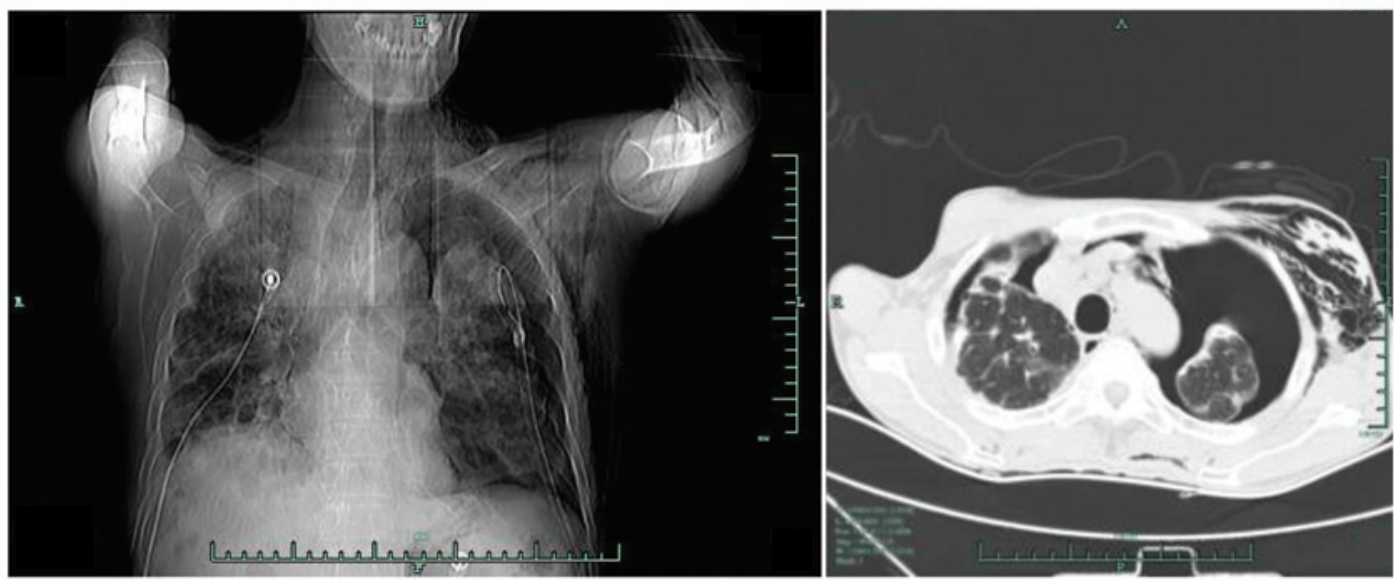

B

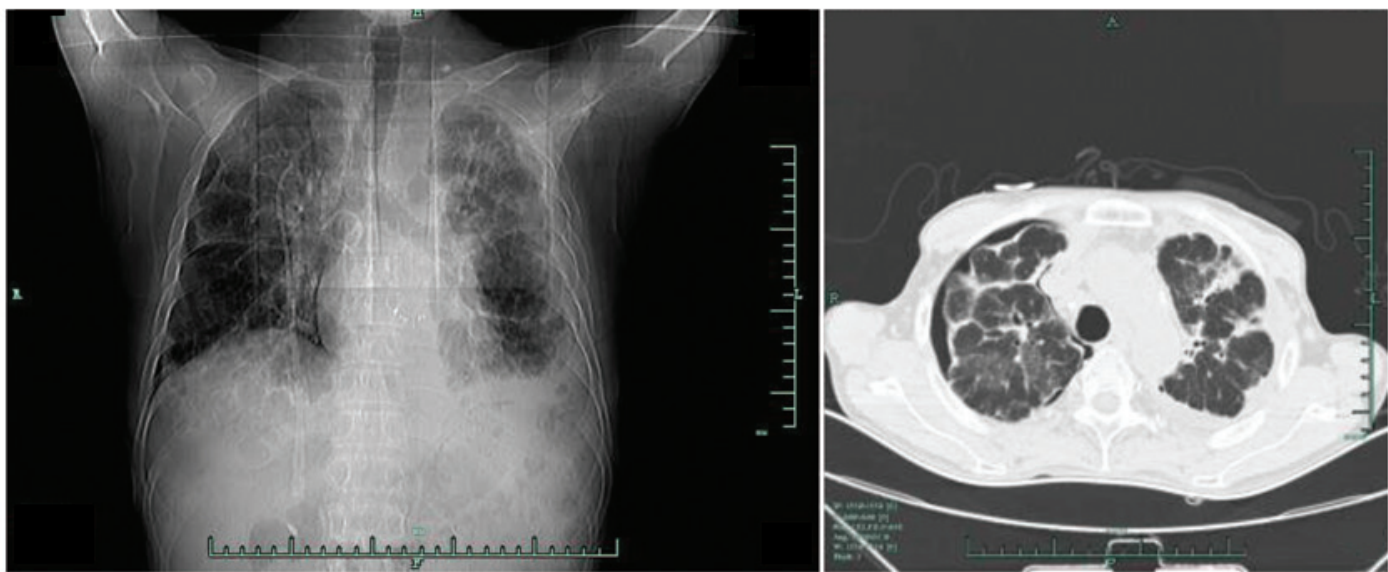

C

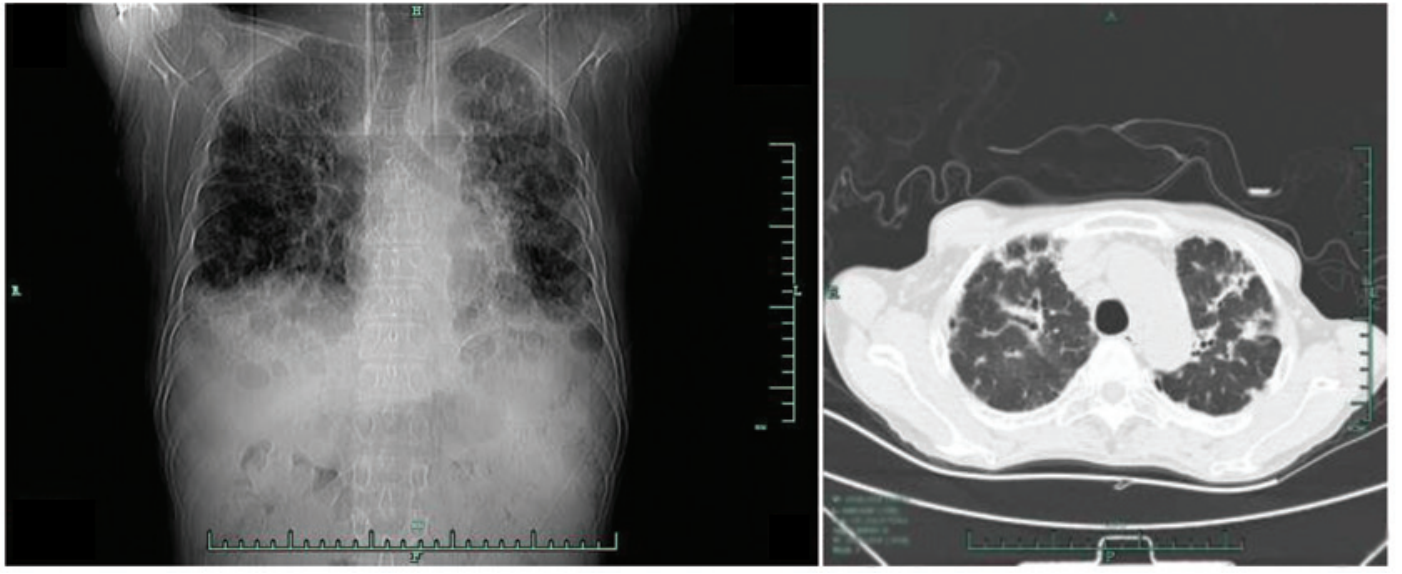

Figure 2. Computed tomography of the lungs demonstrating: (A) Interstitial inflammation of each lung, with the right lung compressed by $5 \%$ and the left lung compressed by $60 \%$; (B) interstitial inflammation of each lung, with the left lung compressed by $20 \%$; and (C) diffusive frosted glass-like changes in the pulmonary tissues and no evidence of a pneumothorax.
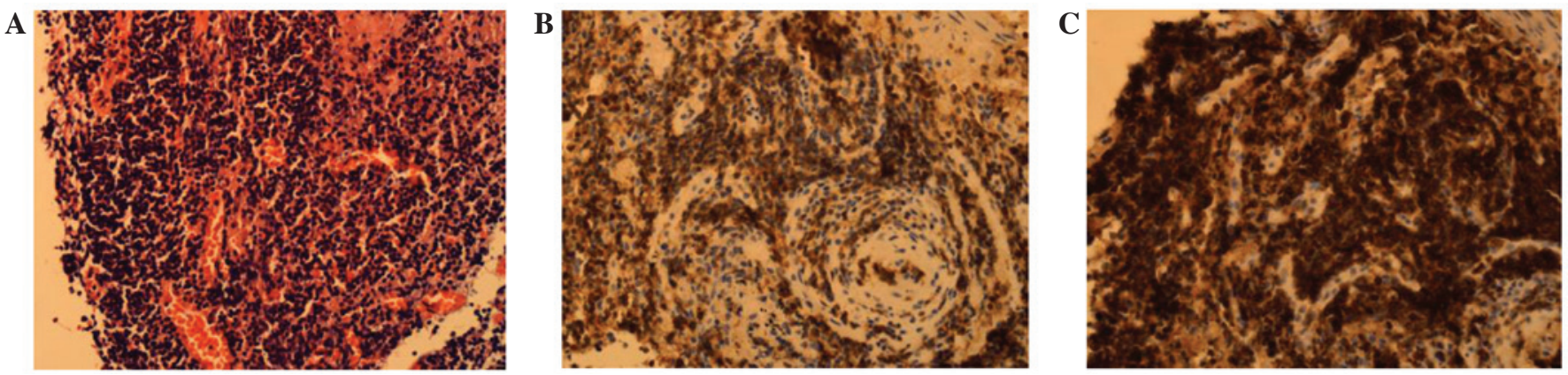

Figure 3. Histopathological examination of the fiber bronchoscopy biopsy. (A) Hyperplastic, small round tumor cells (staining, hematoxylin and eosin) (B) Tumor cell CD3 expression (EnVision method). (C) Tumor cell CD43 expression (EnVision method). Magnification, x400. CD, cluster of differentiation. 

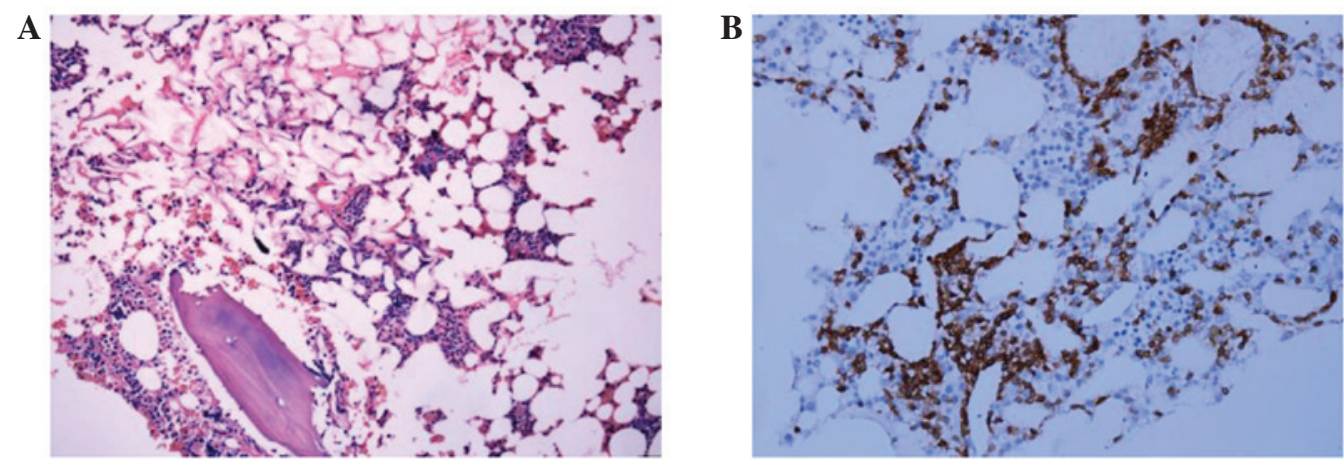

Figure 4. Pathological examination of the bone marrow tissue. (A) Trabecular scattered infiltration of tumor cells (staining, hematoxylin and eosin); and (B) Tumor cell expressing cluster of differentiation 3 (EnVision method). Magnification, x200.

blood culture, C-reactive protein, endotoxin and procalcitonin levels were normal. To test for tuberculosis, a T-SPOT ${ }^{\circledR}$.TB test (Shanghai Fosun Long March Medical Science Co.,Ltd., Shanghai, China) and purified protein derivative (PPD) test (Chengdu Institute of Biological Products Co., Ltd., Chengdu, China) were performed; the T-SPOT ${ }^{\circledR}$.TB test yielded positive results, whilst the PPD test results were negative.

The EnVision two-step staining method with 3,3'-diaminobenzidine (DAB) was used for immunohistochemical analysis throughout the case. The primary antibodies used were monoclonal mouse antibodies (anti-CD2, clone AB75, 1:300 dilution, catalog no. M7309; anti-CD3, clone F7.2.38, 1:600 dilution, catalog no. A0452; anti-CD4, clone 4B12, 1:400 dilution, catalog no. M7310; anti-CD5, clone 4C7, 1:100 dilution, catalog no. M3641; and anti-CD8, clone C8/144B, 1:50 dilution, catalog no. M7103; anti-CD43, clone DF-T1, 1:2,500 dilution, catalog no. M0786; anti-CD20, clone L26, 1:200 dilution, catalog no. M0755; anti-CD10, clone 56C6, 1:50 dilution, catalog no. M7308; anti-CD30, clone Ber-H2, 1:1200 dilution, catalog no. M07G1; anti-CD56, clone 123C3, 1:400 dilution, catalog no. M7304; anti-Ki-67, clone MIB-1, 1:2,000 dilution, catalog no. M7240; and anti-CD45, clone 2B11+PD7/26; 1:4,000 dilution, catalog no. M0701) purchased from Dako (Carpinteria, CA, USA), and a rabbit monoclonal antibody (anti-PAX5, clone SP34, 1:200 dilution, catalog no. GT209629) and a mouse monoclonal antibody (anti-TDT, clone SEN28, 1:100 dilution, catalog no. GT202G29) purchased from Gene Tech Shanghai Co., Ltd. (Shanghai, China).

A fiber bronchoscopy biopsy sample was collected on July 2, 2013. The sample was fixed in formalin, dehydrated, cut into $3-\mu \mathrm{m}$ thick sections and then embedded in paraffin prior to hematoxylin and eosin staining. Histopathological analysis indicated the presence of chronic mucosal inflammation accompanied by mild to moderate small lymphoid tissue hyperplasia. Acid-fast and periodic acid-Schiff staining was negative. Immunoenzymatic labeling of the tissue demonstrated that it was positive for cluster of differentiation (CD)3 and CD43, and negative for CD20 and PAX-5, with a Ki-67 index of 5\%. As a result, the pathologist suspected a diagnosis of non-Hodgkin lymphoma (NHL) (Fig. 3). The patient subsequently underwent a number of examinations to validate this possibility. An abdominal B-mode ultrasound indicated that the liver was of a normal size and that the spleen had a thickness

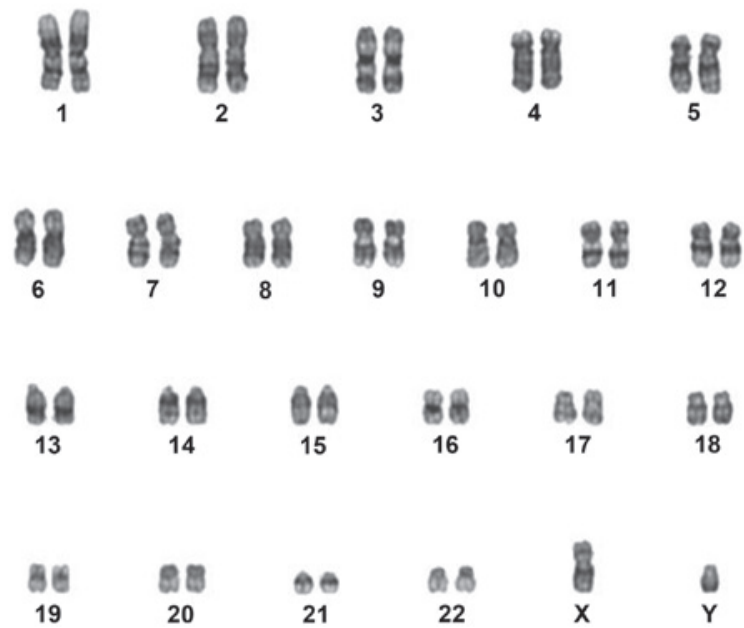

Figure 5. Karyotype: 46,XY. Banding method, G band.

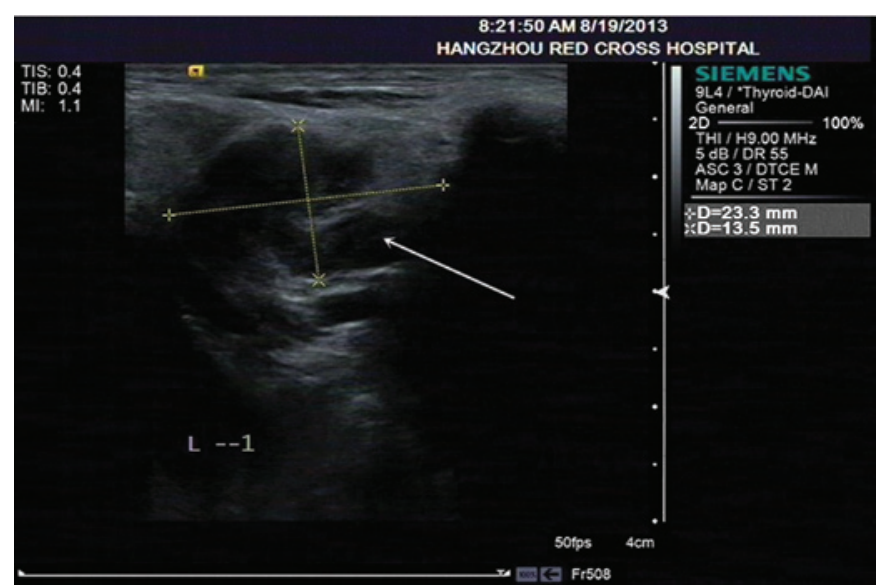

Figure 6. B-mode ultrasound of the lymph node in the left of the neck measuring $2.3 \times 1.3 \mathrm{~cm}$; the scan also shows unclear hilar lymph nodes with non-rich color signals (arrow).

of $\sim 4.6 \mathrm{~cm}$. Several lymph gland resounds were observed adjacent to the hepatic hilar region and pancreas and surrounding the abdominal aorta (the largest being $2.7 \times 1.3 \mathrm{~cm}$ ), in addition to an unclear hilar lymph node. A bone marrow sample was obtained, and then fixed in Bouin solution for $24 \mathrm{~h}$ and decalcified using nitrate acid for $12 \mathrm{~h}$. The sample was then washed with water for $30 \mathrm{~min}$ to remove all the excess chemicals. The 

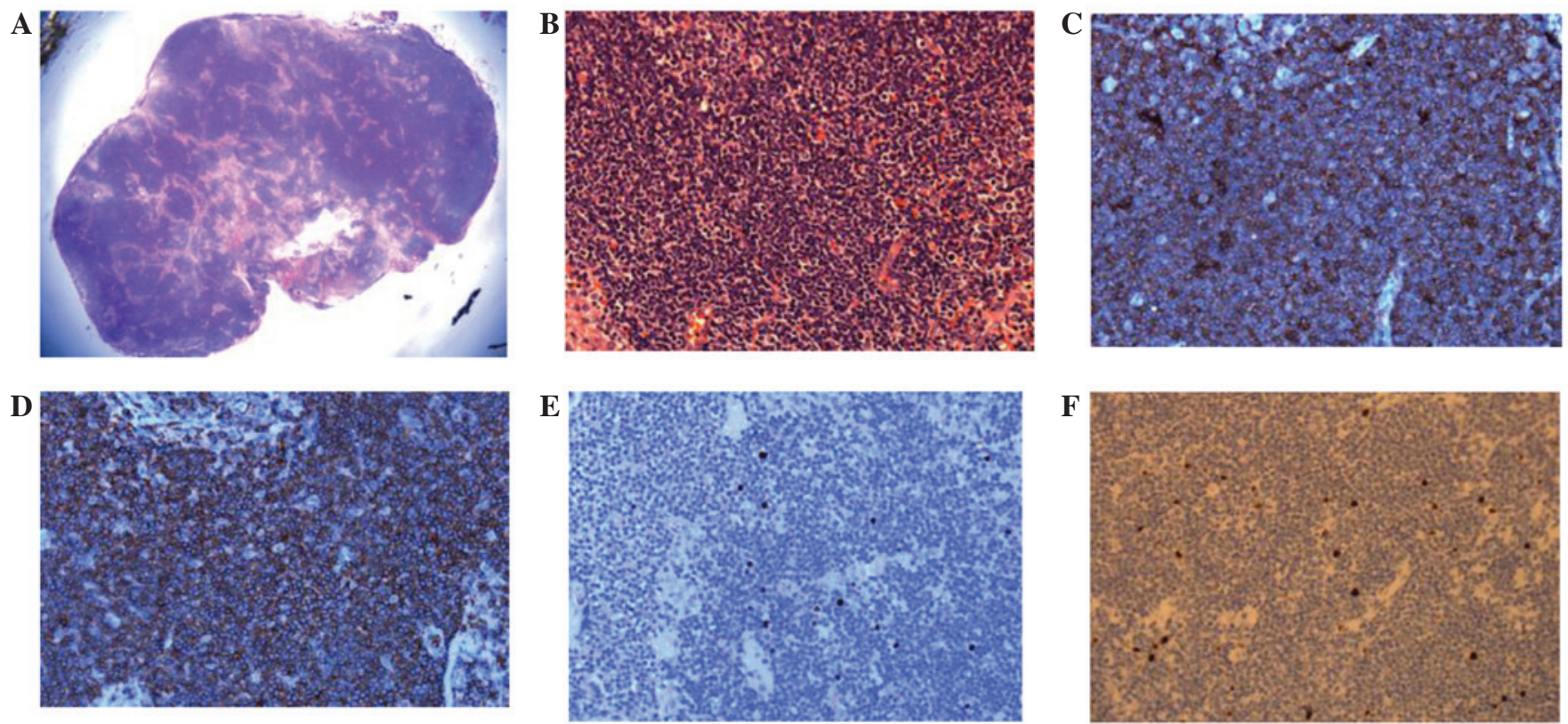

Figure 7. Analysis of the lymph node biopsy. (A) Lymph node structure (staining, HE; magnification, x20). (B) Damaged lymph node structure, with diffusive distribution of small to medium-sized tumor cells, and tumor cell infiltration of the lymph node capsule and surrounding soft tissues (staining, HE). Tumor cell (C) CD3 and (D) CD43 expression (EnVision method); (E) tumor cell Epstein-Barr virus hybridization in situ; and (F) Ki-67 index of 5\%. Magnification, $\mathrm{x} 400$. HE, hematoxylin and eosin; CD, cluster of differentiation.

Control Gene Tube:

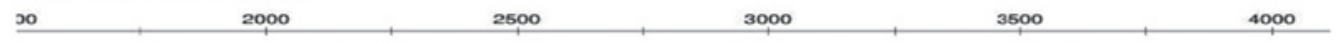

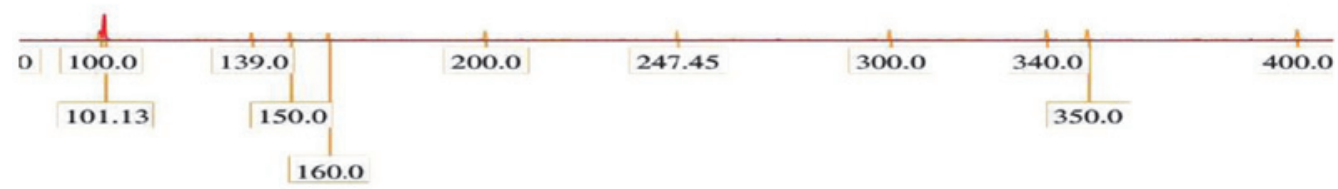

TCRBC: Fluorescent black

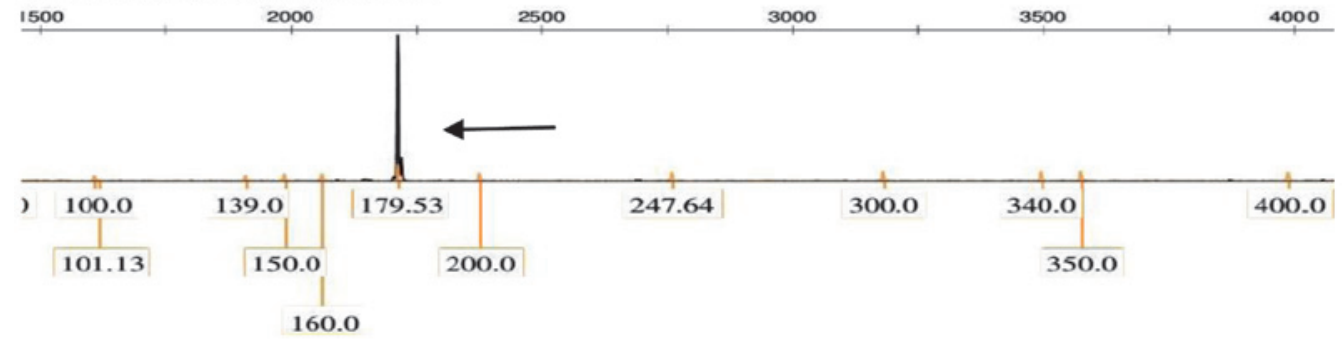

Figure 8. Detection of T cell receptor gene rearrangement. Multiple polymerase chain reaction + GeneScan ${ }^{\circledR}$ software $($ Applied Biosystems; Thermo Fisher Scientific, Inc., Waltham, MA, USA) segment analysis was positive (arrow, positive result). TCRBC, T-cell receptor $\beta$-chain.

sample was fixed in formalin, dehydrated, cut into $3-\mu \mathrm{m}$ thick sections and then embedded in paraffin prior to hematoxylin and eosin or DAB staining. Bone marrow analysis detected an increased proportion of mature lymphocytes, and bone marrow cell immunophenotyping (with threshold analysis set on a CD45/side scatter point diagram) demonstrated that lymphocytes accounted for $\sim 31 \%$ of karyocytes $\left(\mathrm{CD} 3^{+} \mathrm{CD} 4{ }^{-} \mathrm{CD} 8{ }^{-}\right.$cells accounted for $\sim 55.16 \%$ of lymphocytes). Immunophenotyping also indicated that human leukocyte antigen-DR, CD2, CD20 and T-cell receptor (TCR $\alpha / \beta$ were positively expressed, whilst CD5 and CD7 were partially expressed; therefore, the most likely diagnosis was abnormal $\mathrm{T}$ cell lymphocyte hyperplasia. The bone marrow biopsy demonstrated low proliferation of the hematopoietic tissues (accounting for $\sim 40 \%$ of the bone bone marrow), a decreased myeloid:erythroid ratio, scattered immature granulocytes, proerythrocyte clusters, scattered megakaryocytes and interstitial scattered $\mathrm{CD}^{+}$lymphocytes. No abnormalities were noted during histopathological analysis of the bone marrow tissue (Fig. 4), and the chromosomes exhibited normal karyotypes (Fig. 5). Therefore, the patient was transferred to the Department of Hematology. Physical examination indicated enlargement of the lymph nodes in the 
neck; a B-mode ultrasound (ACUSON S2000 ${ }^{\mathrm{TM}}$; Siemens AG) demonstrated that the lymph node located in the left side of the neck was $2.3 \times 1.3 \mathrm{~cm}$ in size, and presented with an unclear hilar lymph node and non-rich color signals (Fig. 6).

The patient underwent a cervical lymph node biopsy in the left of the neck under local anesthesia on August 30, 2013. The sample was prepared as aforementioned for the bronchoscopy sample. Post-operative pathological examination indicated that the normal structure of the lymph node had disappeared. Additionally, mild to moderate-sized diffusive, irregular leukomonocytes were observed; the envelope and extranodal soft tissue had been infiltrated, and hyperplasia of the endothelial vein had been noted, alongside a small number of plasmocytes. Immunoenzymatic labeling indicated that $\mathrm{CD} 2, \mathrm{CD} 3, \mathrm{CD}$, CD8 and CD43 were positive, whilst CD20, CD10, PAX5, CD30, TDT and CD56 were negative, with a Ki-67 index of $5 \%$. Due to the combination of these immunohistochemical results, a diagnosis of PTCL-u was suspected (Fig. 7). TCR gene rearrangement in the lymph node tissues was positive (Fig. 8), and immunoglobulin gene rearrangement was negative. Re-examination of bone marrow cell immunophenotyping was also consistent with a possible diagnosis of T-cell lymphoma $\left(\mathrm{CD}^{-} \mathrm{CD}^{+}{ }^{+} \mathrm{CD} 8^{+}\right)$. Epstein-Barr viral antibody was negative. As the patient presented with symptoms that included night sweats, a fever and a reduction in body weight during hospitalization, the final diagnosis was confirmed as PTCL-u stage IVB (20), and the pneumothorax was considered to have occurred as a result of PTCL-u pulmonary infiltration.

On September 9, 2013, the patient received the first cycle of CHOP chemotherapy [cyclophosphamide (CTX), $750 \mathrm{mg} / \mathrm{m}^{2}$, day 1; vindesine (VDS), $4 \mathrm{mg}$, day 1; epi-adriamycin (ADM), $50 \mathrm{mg}$, day 1; dexamethasone (DXM), $15 \mathrm{mg}$, days 1-5]. The second T-SPOT.TB test was positive and, following a group consultation by the Tuberculosis Department, it was advised that the patient should begin preventative antituberculosis therapy (rifampin, $0.45 \mathrm{~g} / \mathrm{day} /$ adult $\mathrm{qd}$; isoniazide, $0.3 \mathrm{~g}$ /day/adult qd). On October 16, 2013, the patient received a second cycle of CHOP chemotherapy (CTX, $1.0 \mathrm{~g}$, day 1; VDS, $4 \mathrm{mg}$, day 1; epi-ADM, $60 \mathrm{mg}$, day 1; DXM, $15 \mathrm{mg}$, days 1-5). Following treatment, the symptoms of fever and night sweats were alleviated, and body weight increased. A further CT scan of the chest on December 3, 2013, showed that the lung tissue exhibited an appearance similar to diffused ground glass, and high density patches were observed with shadows in the form of accumulated strips. There was no obvious evidence of stenosis in the bronchia or trachea, and no sign of pleural effusion or pneumothorax (Fig. 2C). In addition, several enlarged lymph nodes were observed in the mediastinum. Although the disease had evidently alleviated following chemotherapy, the patient withdrew from further treatment due to financial difficulty, and succumbed to the disease 6 months later.

\section{Discussion}

The development of pneumothorax is common, with the majority of cases occurring as a result of pulmonary infection, tuberculosis or bronchogenic carcinoma (21). On rare occasions, pneumothorax may also be induced by pulmonary lymphoma, the majority of these being B-cell lymphoma (21).
When diagnosing a pulmonary lymphoma, it is important to first determine whether it is primary or secondary (22). Primary pulmonary lymphomas originate from bronchial submucosal and arteriovenous lymphatic tissues. A definitive diagnosis should include the following: i) Clear histopathological diagnosis; ii) pathological changes limited to the pulmonary area, with or without mediastinal and hilar lymph node implications; and iii) 3 months after diagnosis, absence of lymphoma in the tissues or organs outside the lungs and bronchi. Primary pulmonary lymphoma is particularly rare, accounting for $<1 \%$ of all pulmonary tumors and 3-4\% of extranodal lymphoma cases (15). The average age of primary pulmonary lymphoma development is $60-70$ years, and this type of disease comprises mucosa-associated B-cell lymphoma, diffusive B-cell lymphoma, mantle cell lymphoma and follicular lymphoma (21). There are two pathological types of pulmonary lymphoma, Hodgkin's lymphoma and NHL, with NHL occurring more prevalently (23). Clinical symptoms may include coughing, expectoration, blood-stained sputum, fever, chest pain and chest oppression. The disease may also manifest differently by affecting various areas of the body, including the lungs, the alveolar septum and the pleural area, identified by imaging analysis (24). The pathological changes commonly observed inside the lungs predominantly affect the pulmonary interstitium and bronchial submucosal tissues, in addition to the bronchial wall (primarily the extra-wall pulmonary interstitium, such that the bronchial lumen remains unobstructed or experiences only slight stenosis) (25). Bronchial submucosal lymphoma involvement may form inner lumen nodular protrusions, or its growth around the bronchial wall may result in limited or extensive bronchial lumen narrowing, or even complete luminal obstruction complicated by pulmonary consolidation and atelectasis (25). When the alveolar septum is affected, the pulmonary septum initially thickens and, as the disease progresses, the alveolar space gradually becomes smaller or completely obstructed (25). The invasions of the pleura is manifested as pleural thickening, patchy infiltrates or nodules, which tend to be distributed with a lack of aggregation (25).

Secondary pulmonary lymphoma is a form of systemic lymphoma (22). In addition to the common symptoms, including coughing, expectoration, hemoptysis, chest pain and pleural effusion, which are clinically manifested by corresponding respiratory, secondary pulmonary lymphoma also presents with systemic superficial lymph node enlargement and/or B symptoms (22). The imaging characteristics associated with these symptoms are similar to those of pneumonia and tuberculosis, and may therefore lead to misdiagnosis (22).

Primary and secondary pulmonary lymphomas present with multiple imaging manifestations; therefore a comprehensive analysis of the clinical pathology, vital signs and symptoms is required to distinguish between the two (22). In the present case, analysis of the lymph node biopsy resulted in the diagnosis of PTCL-u. The patient was experiencing a fever, night sweats, decreased body weight and a pneumothorax and, as the disease was not limited to the lungs, this lead to the induction of hepatosplenomegaly. As a result, the patient was diagnosed with pneumothorax induced by PTCL-u pulmonary infiltration. 
Regarding the present case, there are a number of mechanisms that may explain how pulmonary lymphoma induced a pneumothorax. Firstly, it may occur as a result of lymphoma cells invading the visceral pleura, or by necrosis exposing the bronchium leading to air leakage (26). Secondly, metastasis may obstruct the surrounding airway, resulting in obstructive emphysema and unstable pulmonary bullae, inducing pneumothorax as a secondary pulmonary disease (26). The present case was considered to occur due to a combination of comprehensive factors. Therefore, to further confirm the underlying mechanisms, pathological methods should be utilized to examine a direct association between the pleura rupture site and the pulmonary lymphoma.

PTCL accounts for 5-15\% of all NHL cases and exhibits marked heterogeneity (12). PTCL-u is the most prevalent form of PTCL and occurs most commonly in the elderly. The disease exhibits high degrees of invasion and malignancy, and often affects the systemic lymph nodes and extranodal sites (typically invading the spleen, skin, digestive tract and bone marrow) (11). As the lungs contain rich lymphatic tissues, PTCL-u should theoretically infiltrate these; however, infiltration of PTCL-u to the lungs is extremely rare (27). Wang et al (28) reported a rare case of cough-variant asthma secondary to angioimmunoblastic T-cell lymphoma; however, to date, there have been no reports of PTCL-u occurring with pneumothorax as the primary manifestation.

The definitive diagnosis of PTCL-u depends upon the pathological analysis of biopsy tissue, with typical manifestations including the dispersive distribution of oncocytes, structural damage of lymph nodes, inflammatory polymorphic background of lymphocytes, eosinophil granulocytes, plasmocytes and a large number of epithelioid tissue cells (27). Oncocytes exist in a number of different forms and may be small, medium or large cells. However, in the majority of cases, the cells are medium to large in size, and have a polymorphic and irregular nucleus with vesicular chromatin and an obvious nucleolus, and frequently form mitotic figures (27). Transparent cells or Reed-Sternberg-like cells are always present, but not true Reed-Sternberg cells. A limited number of cases report of tissue biopsies predominantly containing small lymphocytes with irregular nuclei. There are also increased small vessels, endothelial cell hypertrophy and branching vessels. PTCL-u expresses $\mathrm{T}$ cell-associated antigens, with $\mathrm{CD}^{+}$serving as a reliable marker for this mechanism, whilst CD45RO and $\mathrm{CD} 43$, which are not specific $\mathrm{T}$ cell-associated antigens, are also expressed. In the majority of cases, lymph nodes have been identified as $\mathrm{CD}^{+}{ }^{+} \mathrm{CD} 8^{-}$(29). Therefore, the diagnosis of lymphoma primarily depends on biopsy of the entire lymph nodes, and clear structure of the lymphocytes and associated immunophenotyping is required (27). In the present case, the lymph node sample from the left of the neck was of good quality and conformed to the diagnostic requirements; however, the bronchial mucosa sample was poor and did not reach the diagnostic requirements for lymphoma. Therefore, the pathological analysis of the lymph node was used to confirm the diagnosis of PTCL-u, and flow cytometry indicated T lymphocyte proliferation. Taking into consideration the clinical manifestations of the present case, a diagnosis of PTCL-u was apparent, and the $\mathrm{CD} 20^{+}$lymphocytes present in the bronchial mucosa were considered to demonstrate increased reactivity.
At present, there are no standard guidelines in place for the treatment of PTCL-u, with therapy primarily based on the CHOP chemotherapy regimen. The disease-free and overall survival times of patients with PTCL-u following conventional CHOP treatment are markedly lower than those of patients with B-cell lymphoma $(30,31)$. Novel therapeutic approaches aiming to improve upon the short-term and long-term efficacy of the currently available PTCL-u treatments are being investigated at present. For example, applications of hematopoietic stem cell transplantation, lenalidomide and cytarabine derivatives (32-34) all demonstrate efficacy; however, further clinical studies are required to determine the safest and most reliable treatment. In the present case, following two cycles of CHOP regimen, the body temperature and weight of the patient returned close to normal, night sweats ceased and CT imaging of the chest revealed no evidence of pneumothorax manifestations. A further chemotherapy regimen was considered (L-asparaginase, etoposide and bendamustine); however, the patient withdrew from treatment due to financial difficulty. A relapse was predicted, and the patient succumbed to the disease 6 months later.

In conclusion, as pneumothorax is a particularly common clinical disease, when forming a diagnosis, physicians should pay attention to the medical history of the patient, the clinical symptoms and the results of physical examination. If the pneumothorax-associated symptoms cannot be explained by a common disease, the possibility of pulmonary lymphoma should be taken into consideration, and the conduction of a biopsy followed by pathological analysis may prevent misdiagnosis. The treatment of PTCL-u remains a challenge in the clinical setting, as the disease is commonly resistant to chemotherapy and is prone to relapse. Stem cell transplantation may be considered as a preferred therapeutic approach for young patients; however, the prognosis is commonly poor. There are currently no existing unified treatment guidelines for PTCL-u. As the amount of research in this area increases, it is anticipated that an effective treatment may be developed in the future.

\section{References}

1. Vose $\mathrm{J}$, Armitage $\mathrm{J}$ and Weisenburger; International T-Cell Lymphoma Project: International peripheral T-cell and natural killer/T-cell lymphoma study: Pathology findings and clinical outcomes. J Clin Oncol 26: 4124-4130, 2008.

2. Federico M, Rudiger T, Bellei M, Nathwani BN, Luminari S, Coiffier B, Harris NL, Jaffe ES, Pileri SA, Savage KJ, et al: Clinicopathologic characteristics of angioimmunoblastic T-cell lymphoma: Analysis of the international peripheral T-cell lymphoma project. J Clin Oncol 31: 240-246, 2013.

3. Lunning MA, Moskowitz AJ and Horwitz S: Strategies for relapsed peripheral T-cell lymphoma: The tail that wags the curve. J Clin Oncol 31: 1922-1927, 2013.

4. Schmitz N, Trümper L, Ziepert M, Nickelsen M, Ho AD, Metzner B, Peter N, Loeffler M, Rosenwald A and Pfreundschuh M: Treatment and prognosis of mature T-cell and NK-cell lymphoma: An analysis of patients with T-cell lymphoma treated in studies of the German High-Grade Non-Hodgkin Lymphoma Study Group. Blood 116: 3418-3425, 2010.

5. Rodríguez J, Conde E, Gutiérrez A, Arranz R, León A, Marín J, Bendandi M, Albo C and Caballero MD: The results of consolidation with autologous stem-cell transplantation in patients with peripheral T-cell lymphoma (PTCL) in first complete remission: The Spanish Lymphoma and Autologous Transplantation Group experience. Ann Oncol 18: 652-657, 2007. 
6. Feyler S, Prince HM, Pearce R, Towlson K, Nivison-Smith I, Schey S, Gibson J, Patton N, Bradstock K, Marks DI and Cook G: The role of high-dose therapy and stem cell rescue in the management of T-cell malignant lymphomas: A BSBMT and ABMTRR study. Bone Marrow Transplant 40: 443-450, 2007.

7. Moskowitz AJ, Lunning MA and Horwitz SM: How I treat the peripheral T-cell lymphomas. Blood 123: 2636-2644, 2014.

8. Savage KJ, Chhanabhai M, Gascoyne RD and Connors JM: Characterization of peripheral T-cell lymphomas in a single North American institution by the WHO classification. Ann Oncol 15: 1467-1475, 2004.

9. Escalón MP, Liu NS, Yang Y, Hess M, Walker PL, Smith TL and Dang NH: Prognostic factors and treatment of patients with T-cell non-Hodgkin lymphoma: The M. D. Anderson Cancer Center experience. Cancer 103: 2091-2098, 2005.

10. Barbarotta L and Hurley K: Romidepsin for the treatment of peripheral T-cell lymphoma. J Adv Pract Oncol 6: 22-36, 2015.

11. Bekkenk MW, Vermeer MH, Jansen PM, van Marion AM, Canninga-van Dijk MR, Kluin PM, Geerts ML, Meijer CJ and Willemze R: Peripheral T-cell lymphomas unspecified presenting in the skin: Analysis of prognostic factors in a group of 82 patients. Blood 102: 2213-2219, 2003.

12. Lee HJ, Im JG, Goo JM, Kim KW, Choi BI, Chang KH, Han JK and Han MH: Peripheral T-cell lymphoma: Spectrum of imaging findings with clinical and pathologic features. Radiographics 23 : 7-26, 2003

13. Salazar AJ, Aguirre DA, Ocampo J, Camacho JC and Díaz XA: Evaluation of three pneumothorax size quantification methods on digitized chest X-ray films using medical-grade grayscale and consumer-grade color displays. J Digit Imaging 27: 280-286, 2014

14. Luh SP: Review: Diagnosis and treatment of primary spontaneous pneumothorax. J Zhejiang Univ Sci B 11: 735-744, 2010

15. Noppen M and de Keukeleire T: Pneumothorax. Respiration 76 121-127, 2008

16. Sahn SA and Heffner JE: Spontaneous pneumothorax. N Engl J Med 342: 868-874, 2000.

17. Hobbs BD, Foreman MG, Bowler R, Jacobson F, Make BJ, Castaldi PJ, San José Estépar R, Silverman EK and Hersh CP COPDGene Investigators: Pneumothorax risk factors in smokers with and without chronic obstructive pulmonary disease. Ann Am Thorac Soc 11: 1387-1394, 2014.

18. Schmidt-Horlohé N, Rudig L, Azvedo CT and Habekost M: Fulminant unilateral pulmonary edema after insertion of a chest tube: A complication after a primary spontaneous pneumothorax. Dtsch Arztebl Int 105: 878-881, 2008.

19. Matano S, Satoh S, Sugiguchi S and Sugimoto T: Pneumothorax associated with malignant lymphoma. Intern Med 49: $2337-2339,2010$

20. Parissis H: Forty years literature review of primary lung lymphoma. J Cardiothoracic Surg 6: 23, 2011.

21. Cadranel J, Wislez M and Antoine M: Primary pulmonary lymphoma. Eur Respir J 20: 750-762, 2002.
22. Niu X, Hu H, Gao J, Nie Y, Zhao W, Xu H, Bai X and Chen L: A clinical analysis of 40 cases of primary and secondary pulmonary lymphoma. Zhonghua Jie He He Hu Xi Za Zhi 37: 502-506, 2014 (In Chinese)

23. Zhu L and Zhang J: Pulmonary malignant lymphoma: A clinicopathological analysis of 16 cases. J Diagn Pathol 20: 740-743, 2013.

24. Zhang ZY, Wu XH and Shi YX: CT study of pulmonary lymphoma comparison with X-ray. Clin Med J China 8: 26-28, 2001.

25. Hare SS, Souza CA, Bain G, Seely JM, Frcpc, Gomes MM and Quigley M: The radiological spectrum of pulmonary lymphoproliferative disease. Br J Radiol 85: 848-864, 2012.

26. Shan Y and Zhou Y: Recent research of pathogenesis and causes of recurrence of primary spontaneous pneumothorax. Surg Res New Technique 4: 52-56, 2015.

27. Went P, Agostinelli C, Gallamini A, Piccaluga PP, Ascani S, Sabattini E, Bacci F, Falini B, Motta T, Paulli M, et al: Marker expression in peripheral T-cell lymphoma: A proposed clinical-pathologic prognostic score. J Clin Oncol 24: 2472-2479, 2006.

28. Wang XY, Xia GG, Zhang YJ, Zhang B and Zhang PJ: Angioimmunoblastic T-cell lymphoma: A case report and review of literature. Int J Respir 32 6: 91-94, 2012 (In Chinese).

29. Zhe W, Xie YQ and Zhu J: The advance in diagnosis and treatment of peripheral T-cell lymphoma-unspecified. Chin J Clin Oncol 33: 476-480, 2006.

30. Morabito F, Gallamini A, Stelitano C, Callea V, Guglielmi C, Neri S, Lazzaro A, Orsucci L, Ilariucci F, Sacchi S, et al: Clinical relevance of immunophenotype in a retrospective comparative study of 297 peripheral T-cell lymphomas, unspecified, and 496 diffuse large B-cell lymphomas: Experience of the Intergruppo Italiano Linformi. Cancer 101: 1601-1608, 2004.

31. Intragumtornchai $\mathrm{T}$, Rotnakkarin $\mathrm{P}$, Sutcharitchan $\mathrm{P}$ and Wannagrairoj P: Prognostic significance of the immunophenotype versus the International Prognostic Index in aggressive non-Hodgk'n/s lymphoma. Clin Lymphoma 4: 52-55, 2003.

32. Gui L, Shi YK, He XH, Lei YH, Zhang HZ, Han XH, Zhou SY, Liu P, Yang JL, Dong M, et al: High-dose therapy and autologous stem cell transplantation in peripheral T-cell lymphoma: Treatment outcome and prognostic factor analysis. Int J Hematol 99: 69-78, 2014.

33. Hopfinger G, Nösslinger T, Lang A, Linkesch W, Melchardt T, Weiss L, Egle A and Greil R: Lenalidomide in combination with vorinostat and dexamethasone for the treatment of relapsed/refractory peripheral T cell lymphoma (PTCL): Report of a phase I/II trial. Ann Hematol 93: 459-462, 2014.

34. Evens AM, Rosen ST, Helenowski I, Kline J, Larsen A, Colvin J, Winter JN, van Besien KM, Gordon LI and Smith SM: A phase I/II trial of bortezomib combined concurrently with gemcitabine for relapsed or refractory DLBCL and peripheral T-cell lymphomas. Br J Haematol 163: 55-61, 2013. 